\title{
Interest Rate Term Structure Decomposition: An Axiomatic Structural Approach
}

\author{
Brian Barnard ${ }^{1}$ \\ ${ }^{1}$ Wits Business School, University of the Witwatersrand (WITS), South Africa \\ Correspondence: Brian Barnard, Wits Business School, University of the Witwatersrand (WITS), South Africa.
}

Received: October 6, 2018

Accepted: December 11, 2018

Available online: December 14, 2018

doi:10.11114/aef.v6i1.1907

URL: https://doi.org/10.11114/aef.v6i1.1907

\begin{abstract}
The paper examines an axiomatic structural approach to term structure decomposition. From this perspective, term structure decomposition is modelled as an non-parsimonious optimization problem, with the structure delineated by constraints related to the likely attributes thereof, rather than by a linear combination of splines or functions. The motivation for the model lies in its perceived flexibility or power. Also, the model is seen as a likely candidate to implement issue-level term structure decomposition. Consequently, issue-level term structure decomposition is also briefly introduced. The power of the model is tested on a simulated and market sample. Even though it may go against notions of structure smoothness, the relationship or correlation between structure smoothness, goodness of fit, and systematic/ unsystematic risk is also touched on.
\end{abstract}

Keywords: term structure decomposition, optimization, market efficiency

\section{Introduction}

The use of forward interest rates has long been standard in financial analysis, for instance in pricing new financial instruments and in discovering arbitrage possibilities (Svensson, 1994). Bolder and Gusba (2002) note the fundamental aspect and importance of risk-free interest rates: In the world of fixed-income, it is difficult to find a more fundamental object than a riskless pure discount bond or, as it is equivalently called, a zero-coupon bond. This is because the price of a pure discount bond represents the current value of one currency paid with complete certainty at some future point in time. Abstracting from the idea of risk premia for longer-term bond holdings, it is essentially a representation of the time value of money. A trivial transformation of the bond price is the rate of return on this simple instrument or, as it is more commonly termed, the zero-coupon interest rate. These building blocks of fixed-income finance are tremendously important for a wide array of different purposes, including bond pricing, discounting future cash flows, pricing fixedincome derivative products, constructing forward interest rates, and determining risk premia associated with holding bonds of different maturities.

With regards to the models used for the extraction of zero-coupon and forward rates from coupon bearing instruments, Marciniak (2006) differentiates between two classes of models: parsimonious (Nelson and Siegel, 1987; Svensson, 1994) and polynomial based models (McCulloch, 1971; McCulloch, 1975; Fisher et al, 1994; Waggoner, 1997; Anderson and Sleath, 2001). Bolder and Gusba (2002) in turn differentiate between spline-based models (McCulloch, 1971; Fisher et al, 1994; Waggoner, 1997; Anderson and Sleath, 2001) piecewise-cubic polynomial-based approaches and function-based models (Vasicek and Fong, 1981; Li et al, 2001; Svensson, 1994) linear combinations of various functions (exponential and trigonometric). In other words, a linear combination of (exponential) basis functions versus a liner combination of splines.

According to Marciniak (2006), term structure models are characterized by: 1) goodness of fit (flexibility), 2) smoothness, 3) stability of results (robustness to changes in the data), and 4) numerical stability and time of computation. Stability in turn relates to four different properties: 1) robustness to outliers, 2) robustness to minor changes in the data set, 3) (non)uniqueness of estimates, and 4) numerical stability. Both Marciniak (2006) and Bolder and Gusba (2002) point to the trade-off between the flexibility (goodness of fit), smoothness and stability. Excess flexibility leads to loss of smoothness (humps) and to a drastic fall in its stability.

A clear verdict on the model classes are not made. Gimeno and Pineda (2006) argue that parsimonious models enjoy 
widespread use by a number of central banks. Marciniak (2006) states that between parsimonious and polynomial models, B-spline models stabilized with a variable roughness penalty have best overall performance.

Also, both model classes have individual challenges. Marciniak (2006) notes a significant weakness of the Nelson-Siegel model, resulting from its low elasticity, is goodness of fit. When the curve is fitted to an irregular set of data points this can result in relatively large deviations of model values from actually observed rates. Even though the parsimonious model of Svensson (1994) addresses this, it has a limited ability to fit irregular yield curve shapes, a tendency to take extreme values at the short end, and a relatively strong co-dependence of estimates in different even non-neighbouring segments of the yield curve.

Gimeno and Pineda (2006) note that with parsimonious models, like Nelson and Siegel (1987) and Svensson (1994), the high degree of non-linearity of the functions to be optimised, make them very sensitive to the initial values employed. They note the risk of false convergence: the risk of achieving parameters corresponding to a local optimum and not to the global optimum relative to the data. This risk comes from the high level of non-linearity of the functions to be fitted, and in practice is often detected by poor fit with regard to conventional levels, a posteriori. This problem is clearly shown by the high sensitivity of the estimated parameters, and not necessarily of goodness-of-fit, to the initial values used in the implementation of the usual nonlinear optimization algorithms. Similarly, Gauthier and Simonato (2012) note two problems pertaining to the Nelson-Siegel or Svenson approaches. First, different parameter values may lead to similar yield curves. Such a result is possible because the model is estimated from coupon bonds with finite maturities. Over the observed maturities, different parameters can get almost identical yield curve estimates. Secondly, with regards to the optimization problem, the function to optimize is not guaranteed to be convex and may show several local optima.

Svensson (1994) notes that the cubic spline has the well-known disadvantage that estimates of forward rates may be rather unstable, especially at the longest maturity. Also, curve smoothness and goodness of fit are sensitive to the number and location of knots (Marciniak, 2006). Fisher et al (1994) state that, with spline-based models, the choice of both the number and placement of the knot points for the spline creates the potential for ad hoc parameterizations, especially as the spline changes through time. Fisher et al (1994), Bolder and Gusba (2002), and Marciniak (2006) discuss how stabilizers or penalty functions attempt to address this issue. According to Marciniak (2006), the main weakness of polynomial and piecewise polynomial models is their insufficient smoothness and stability. Implied forward rates calculated from polynomial-based discount functions have a tendency to form a highly oscillatory term structure. Moreover, they do not have any asymptotic convergence properties and consequently for maturities beyond the observed spectrum usually take totally unrealistic and non-interpretable (often negative) values. This is directly related to the asymptotic properties of polynomials, which always have infinite boundaries.

Next, an axiomatic structural approach to term structure decomposition is discussed. Given that a strong motivation for such an approach is issue-level term structure decomposition, this is also subsequently delineated. Essentially, the purpose of the study is to show that term structures can be decomposed as an optimization problem, when stipulating constraints that steer the structure. It is not to evaluate the power of the model, viz-a-viz conventional models. Also, the relationship or correlation between systematic/ unsystematic risk and structure smoothness or variance is briefly examined.

\subsection{Term Structure Decomposition as a General Optimization Problem With Constraints to Guide Structure}

The premise that the resultant portfolio term structure optimizes goodness of fit against structure smoothness, may already stipulate sufficient information to decompose a term structure, without necessarily stipulating a set of basis functions or splines. Rather, it may simply be stated as a general optimization problem, by including constraints to guide the structure or smoothness thereof (Boyd and Vandenberghe, 2004).

Such an approach may offer various advantages: it offers greater freedom in terms of sub-structures across intervals, and interval width or span knots associated with spline models. Intervals do not need to be of equal width or predefined number. Thus, from the vantage point of a conventional model, it is less restrictive in terms of the imposed term structure, and it may be seen as including a greater number of basis functions. Therefore, it should have comparable or greater power than conventional models. However, the greatest motivation is its ease of application of issue-level term structure decomposition, as is discussed subsequently.

Given the spot rate $\left(r^{S}\right)$ over the interval from the present to coupon date $t$, the price of an issue $\left(P^{i}\right)$ is given by the sum of its $\mathrm{M}$ discounted coupons (equation 1). Then, given $\mathrm{N}$ bond issues, the coupons of a portfolio $\left(C^{p}\right)$ for the portfolio coupon date $t$, is given by the sum of all issue coupons $\left(C^{i}\right)$ on said coupon date $t$ (equation 2.a). To aid explaination, consider that, by introducing zero coupons, each and every issue can be seen to have a coupon on each portfolio coupon date. Similarly, the portfolio price $\left(P^{p}\right)$ is given by the sum of the issues' prices $\left(P^{i}\right)$ (equation 2.b). A portfolio discount factor $\left(D F^{p}\right)$ is naturally derived from the portfolio spot rate $\left(r^{s p}\right)$ corresponding to the portfolio coupon dates (equation 3 ). The issue price is recast in terms of the $\mathrm{K}$ portfolio discount factors, and portfolio coupon dates (equation 4). The number of portfolio coupon dates $(\mathrm{K})$ is generally greater than or equal to the maximum of the $\mathrm{N}$ issues' number of issue 
coupon dates $\left(M^{i}\right)$.

$$
\begin{gathered}
P_{n}^{i}=\sum_{m=1}^{M_{n}^{i}} C_{n m}^{i} e^{-r_{t_{m}}^{s} t_{m}} ; n \in[1, \ldots, N] \\
C_{t}^{p}=\sum_{n=1}^{N} C_{n t}^{i} \\
P^{p}=\sum_{n=1}^{N} P_{n}^{i} \\
D F_{t}^{p}=e^{-r_{t}^{s p} t^{p}} \\
P^{i}=\sum_{n=1}^{M} C_{n}^{i} e^{-r_{t_{n}}^{s} t_{n}}=\sum_{k=1}^{K} D F_{k}^{p} C_{k}^{i} \\
K \geq \max \left(M_{i}\right) ; i \in[1 ; N]
\end{gathered}
$$

Below, equation set 6 depicts term structure decomposition as an optimization problem. The sum of the residual between issue market price $\left(P^{i m}\right)$ and modelled price $\left(P^{i}\right)$ across all issues is minimized (equation 6.a). An equality constraint is added: the portfolio coupons the amalgamation of issue coupons discounted against the resultant portfolio term structure should equal the portfolio value, given as the sum of the portfolio issues' prices (equation 6.b). In this case, inequality constraints are also added to prevent negative forward rates across the $\mathrm{K}$ portfolio coupon dates (equation 6.c 6.e).

$$
\text { minimize } \sum_{n=1}^{N}\left(P_{n}^{i m}-P_{n}^{i}\right)^{2}
$$

subject to:

$$
\begin{gathered}
P^{p}=\sum_{k=1}^{K} D F_{k}^{p} C_{k}^{p} \\
D F_{1}^{p} \leq 1 \\
D F_{k}^{p} \geq D F_{(k+1)}^{p} ; k \in[1, \ldots, K-2] \\
D F_{K}^{p} \geq 0 \\
V^{p}=\sum_{k=1}^{K-1}\left(\frac{F_{t_{k}^{k+1}}^{p}-F_{t_{k-1}^{k}}^{p}}{t_{k}^{k+1}}\right)^{2} \leq V^{p C}
\end{gathered}
$$




$$
\begin{gathered}
=\sum_{k=1}^{K-1}\left(-1 / t_{k}^{k+1} \cdot 1 / t_{k}^{k+1} \ln \left(D F_{k+1}^{p} / D F_{k}^{p}\right)+1 / t_{k}^{k+1} \cdot 1 / t_{k-1}^{k} \ln \left(D F_{k}^{p} / D F_{k-1}^{p}\right)\right)^{2} \\
=\sum_{k=1}^{K-1}\left(-a \cdot \ln \left(D F_{k+1}^{p}\right)+(a+b) \cdot \ln \left(D F_{k}^{p}\right)-b \cdot \ln \left(D F_{k-1}^{p}\right)\right)^{2} \\
\left(\frac{F_{t_{k}^{k+1}}^{p}-F_{t_{k-1}^{k}}^{p}}{t_{k}^{k+1}}\right)^{2} \leq V^{p p c} ; k \in[1, \ldots, K-1]
\end{gathered}
$$

With regards to the optimal structure to induce, Bolder and Gusba (2002) state that zero-coupon curve smoothness is a relevant criterion for a term-structure estimation model, because overly non-smooth zero-coupon curves are highly oscillatory functions in a model. This implies the occurrence of dramatic swings in rates from one period to the next. Typically, the term structure of interest rates is expected to move gradually across the term-to-maturity spectrum. Dramatic moves, conversely, are not considered reasonable. There is not really a possible economic reason to explain a large difference between the price of a five-year pure discount bond and a five-year-and-one-week pure discount bond.

Marciniak (2006) notes that parsimonious models infer several basic shapes: monotonous increasing or decreasing, humped, or S-shaped. The value of one of the coefficients should correspond to zero-coupon rates for ultra long maturities. At the short end of the curve, the sum of some of the parameter values should be equal to the level of the shortest interest rates. Bolder et al (2004) in turn note the Merrill Lynch exponential spline (MLES) model is strictly based on curve-fitting techniques. That is, it is a strictly mathematical process defined as fitting a continuous function to a set of discretely observed data points. The process of generating the yield curve makes no underlying economic assumptions, nor does it impose any functional form to the yield curve. It models the discount function as a linear combination of exponential basis functions.

In light of this, the primary constraint on the output structure is taken as the requirement that individual rates must grow gradually, with neighbouring or periods of rates not demonstrating dramatic moves. In addition, the short end of the curve may be constrained to conform with immediate term expectations. This may be accomplished by assigning weights to issues and their price residuals, according to their maturities (Bolder and Gusba, 2002; Marciniak, 2006).

Therefore, to constrain variance in the output structure, another constraint is added: the output variance $\left(V^{p}\right)$ may not exceed a certain ceiling $\left(V^{p C}\right)$, defined in advance (equation 6.f). Output variance is based on the portfolio forward $\operatorname{rates}\left(F^{p}\right)$, and is measured as the forward rate growth rates. It can also be argued that another property of the term structure is that the growth rate between any two points would never exceed a certain rate $\left(V^{p p C}\right)($ equation 7$)$. This is incorporated in the constraint on the structure variance sum (equation 6.f) to some extent; stating it as an additional, separate constraint ought to implement it more strictly though. This constraint is not implemented in this study.

A minimum variance solution is sourced to serve as initial solution for the optimization problem. This solution ignores issue price modelling residuals altogether, and instead minimizes the variance. Seeking a single or universal forward rate $\left(f^{u}\right)$ that causes the portfolio coupons discounted at the resultant portfolio discount factors that yields the portfolio value (equation 8), provides such an initial solution.

$$
\begin{gathered}
D F_{1}^{p}=e^{\left(-f^{u} t_{0}^{1}\right)} \\
D F_{n}^{p}=e^{\left(-f^{u} t_{n-1}^{n}\right)} \cdot D F_{n-1}^{p} ; n \in[2, \ldots, K]
\end{gathered}
$$

Thus, in addition to modelling residual as primary objective of the optimization problem, variance as secondary objective is introduced. However, variance is not directly included in the objective function typically done by assigning a weight to it but by modelling it as additional, albeit dynamic, constraint in the form of a predefined ceiling.

The model agrees with conventional models in terms of its primary objective to minimize issue price modelling error (equation 6). However, instead of basing constraints on the acceptable output structure on mathematical functions, it is rather based on the attributes of the structure. Two attributes were considered: the structure has minimum variance, and the growth rate between any two points likely do not exceed a given value.

\subsection{Negative Forward Rates}

The optimization problem above also includes constraints to prevent negative forward rates. The inclusion of such 
constraints is further deliberated, but generally left as an open question.

A number of axioms can be noted with regards to negative forward rates. Hypothetically, a sequence of intervals may contain positive and negative forward rates. Of interest here are instances of interval sequences with negative-only forward rates, or mixed-positive and negative-forward rates. Furthermore, discounting coupons against such a sequence may result in a present value lower or higher than the coupon itself. The determinants of forward rates-simply the determinants of interest rates ${ }^{1}$-are seen as finite; the forward rate of an interval is seen as the sum of the weights of the determinants of forward rates in general. Arguably, even though they may be zero, some of the (weights of the) determinants of forward rates can not be negative-default risk, and tax premiums come to mind; liquidity premiums may perhaps also be added. One determinant that may indeed be negative, is inflation-therefore indicating deflation.

From a risk-neutral perspective, the notion of a negative forward rate may not necessarily imply that investment instruments containing negative forward rates would or should be avoided-even with negative forward rates, purchasing power or value may be preserved, such that the risk-neutral investor is indifferent to negative forward rates. However, investors may have a subjective bias towards negative forward rates, particularly if the negative forward rate implies deflation. Secondly, and more importantly, the investor must be unable to source an alternative investment that can circumvent the negative forward rate over the interval, to be truly indifferent to a negative forward rate over the interval. This may imply investments internal or external to the market.

Technically, trading the appropriate issues should remove negative forward rates from intervals, given that issue prices and their coupons impact the forward rates of individual intervals. However, there must be sound justification to shape intervals' rates through trade. Thus, negative forward rates imply at least basic market efficiency-intervals have corresponding negative forward rates because the market views this as fair price. In this context, basic market efficiency also implies basic consistency of forward rates across intervals.

When justifying negative forward rates against external markets, currency risk may be introduced, perhaps seen as related to liquidity risk. However, this again reverts to basic market efficiency. Triangulation must persist between a) term structures and forward rates as measures of risk, and b) currency exchange rates, as well as currency forwards. Forward rates of term structures must reflect on and correlate with currency forwards to prevent arbitrage due to risk/ exchange rate disequilibrium.

\subsection{Issue-Level Term Structure Decomposition}

Issue-level term structure decomposition implies that a term structure is decomposed for each issue. Furthermore, the portfolio term structure is not directly decomposed, but derived from the issue term structures instead. Its aim is to offer a more direct way to simultaneously capture both the more predominantly unsystematic risk structures of issues, and the more predominantly systematic risk structure of the portfolio, through the mathematical relationship that links the two sets. It allows individual issue term structures to naturally deviate from the portfolio term structure, by allowing for the unsystematic risk components of issues. This should advance the understanding of factors of pricing, in that it permits an analysis and comparison between individual issue term structures and the portfolio term structure, in line with the inquiry by Elton et al (2001), just in a more detailed and elaborate manner.

Issue term structures and the corresponding portfolio term structure are naturally and logically linked. Decomposing issue term structures implies that, for each issue, discounting the issue's coupons at issue-unique discount factors should equal the issue price. In addition, discounting the portfolio coupons at the portfolio discount factors should equal the portfolio price. Overall, two relationships are preserved: First, the portfolio price obtained by discounting the portfolio coupons against the portfolio term structure, must equal the sum of the prices of the $\mathrm{N}$ issues associated with the portfolio, obtained by discounting each issue's coupons against each issue's term structure (equation 11.b). Secondly, and perhaps more importantly, discounting any portfolio coupon at the portfolio discount factor for a specific portfolio coupon date, must equal the sum of the corresponding issue coupons discounted at their specific issue discount factors for that coupon date (equation 9). Thus, the portfolio coupon $\left(C^{p}\right)$ for portfolio coupon date $\mathrm{k}$, discounted at the portfolio discount factor $\left(D F_{k}^{p}\right)$ of date $\mathrm{k}$, should equal the sum of the issue coupons $\left(C_{k}^{i}\right)$ of date $\mathrm{k}$, discounted at the corresponding issue discount factor $\left(D F_{k}^{i}\right)$ of date $\mathrm{k}$. For simplification, the portfolio coupon date, $\mathrm{k}$, an issue coupon and coupon date $I_{m}^{i}$ maps to, is notated as $P_{a[i][m]}$.

$$
D F_{k}^{p} C_{k}^{p}=\sum_{i}^{N}\left(D F_{k}^{i} C_{k}^{i}\right) ; k \in[1, \ldots, K]
$$

1. On the determinants of bond spreads, see for instance Elton et al (2001). 


$$
I_{m}^{i} \rightarrow P_{a[i][m]}
$$

This is captured by the optimization problem equation set 11 . Given $\mathrm{N}$ issues, each with $M^{i}$ coupon dates, the difference between modelled issue prices $P_{n}^{i}$, and market issue prices $P_{n}^{i m}$ is minimized (equation 11.a). An equality constraint may be added to constrain the portfolio price to its actual value (equation 11.b). Inequality constraints are added to prevent negative issue (equation 11.c-e), and portfolio (equation 11.f-h) forward rates. The initial solution of equation 8 would also function in this case.

$$
\operatorname{minimize} \sum_{n=1}^{N}\left(P_{n}^{i m}-P_{n}^{i}\right)^{2}
$$

subject to:

$$
\begin{gathered}
P^{p}=\sum_{k=1}^{K} D F_{k}^{p} C_{k}^{p}=\sum_{i=1}^{N} \sum_{m=1}^{M^{i}} D F_{m}^{i} C_{m}^{i} \\
D F_{1}^{p} \leq 1 \\
D F_{k}^{p} \geq D F_{(k+1)}^{p} ; k \in[2, \ldots, K-1] \\
D F_{K}^{p} \geq 0 \\
D F_{1}^{i} \leq 1 ; i \in[1, \ldots, N] \\
D F_{m}^{i} \geq D F_{(m+1)}^{i} ; m \in\left[2, \ldots, M^{i}-1\right] \\
D F_{M^{i}}^{i} \geq 0
\end{gathered}
$$

In order to constrain the structures of the issue and portfolio term structures, a number of structure axioms are noted. A basic degree of smoothness is expected over individual issues' term structures (equation 12), and this should naturally extend to the portfolio term structure (equation 6.f). In addition, it is expected that issues should have discount factors and forward rates comparable to that of the portfolio for comparable dates (equation 13 to 15). Similarly, issues are expected to have comparable discount factors and forward rates over their overapping coupon intervals (equation 16).

Thus, the variance across the individual issue term structures (equation 12), and the portfolio term structure (equation 6.f) is expected to be minimum. Given that they relate to similar periods in time, the distance between the issue discount factors, and the portfolio discount factors, is expected to be minimum (equation 13). Because the interval lenghts of issues and the portfolio differ, issues' discount factors may be expected to be comparable to all portfolio discount factors falling within the issues' coupon interval (equation 14). This is an extension of the second condition mentioned above (equation 13). Because forward rates ought to be more sensitive, the extension of the second condition can also be rewritten in terms of forward rates (equation 15). Instead of basing it on the portfolio forward rates, it can also be based on the issue forward rates of adjacent issues overlapping with the issue interval (equation 16).

$$
\begin{gathered}
\sum_{i=1}^{N} \sum_{m=1}^{M^{i}-1}\left(\frac{F_{t_{m}^{m+1}}^{i}-F_{t_{m-1}^{m}}^{i}}{t_{m}^{m+1}}\right)^{2} \leq C^{i v} \\
\sum_{i=1}^{N} \sum_{m=1}^{M^{i}-1}\left(-1 / t_{m}^{m+1} \cdot 1 / t_{m}^{m+1} \ln \left(D F_{m+1}^{i} / D F_{m}^{i}\right)+1 / t_{m}^{m+1} \cdot 1 / t_{m-1}^{m} \ln \left(D F_{m}^{i} / D F_{m-1}^{i}\right)\right)^{2} \leq C^{i v}
\end{gathered}
$$




$$
\begin{gathered}
\sum_{i=1}^{N} \sum_{m=1}^{M^{i}-1}\left(-a \cdot \ln \left(D F_{m+1}^{i}\right)+(a+b) \cdot \ln \left(D F_{m}^{i}\right)-b \cdot \ln \left(D F_{m-1}^{i}\right)\right)^{2} \leq C^{i v} \\
\sum_{i=1}^{N} \sum_{m=1}^{M^{i}}\left(D F_{m}^{i}-D F_{a[i][m]}^{p}\right)^{2} \leq C^{i p d} \\
\sum_{i=1}^{N} \sum_{m=1}^{M^{i}} \sum_{j=1}^{J^{i j}}\left(D F_{m}^{i}-D F_{a[i][m][j]}^{p}\right)^{2} \leq C^{i p d} \\
\sum_{i=1}^{N} \sum_{m=1}^{M^{i}} \sum_{j=1}^{J^{i m}}\left(F_{m}^{i}-F_{a[i][m][j]}^{p}\right)^{2} \leq C^{i p f d} \\
\sum_{i=1}^{N} \sum_{m=1}^{M^{i}} \sum_{j=1}^{J^{i m}}\left(-t_{m-1}^{m}\left(\ln \left(D F_{m}^{i}\right)-\right.\right. \\
\left.\left.\ln \left(D F_{m-1}^{i}\right)\right)+t_{j-1}^{j}\left(\ln \left(D F_{a[i][m][j]}^{p}\right)-\ln \left(D F_{a[i][m][j-1]}^{p}\right)\right)\right)^{2} \leq C^{i p f d} \\
\sum_{i=1}^{N} \sum_{m=1}^{M^{i}} \sum_{l=1}^{L^{i m}}\left(F_{m}^{i}-F_{b[i][m][l]}^{i}\right)^{2} \leq C^{i i f d} \\
\sum_{i=1}^{N} \sum_{m=1}^{M^{i}} \sum_{l=1}^{L^{i m}}\left(-t_{m-1}^{m}\left(\ln \left(D F_{m}^{i}\right)-\right.\right. \\
\left.\left.\ln \left(D F_{m-1}^{i}\right)\right)+t_{l-1}^{l}\left(\ln \left(D F_{a[i][m][l]}^{i}\right)-\ln \left(D F_{a[i][m][l-1]}^{i}\right)\right)\right)^{2} \leq C^{i p f d}
\end{gathered}
$$

In the above, $N$ refers to the number of issues, and $M^{i}$ refers to the coupon dates of each issue $i . F_{t_{m}^{m+1}}^{i}$ is the forward rate of an issue $i$ over its coupon interval $t_{m}^{m+1}$. Similarly, $F_{m}^{i}$ is the forward rate of an issue $i$ over its coupon interval demarcated by coupon date $m$ and $m-1 . D F_{m}^{i}$ is the discount factor of issue $i$ for the coupon date $m . D F_{a[i][m]}^{p}$ is the portfolio discount factor corresponding to the coupon date $m$ of issue $i$. Similarly, $D F_{a[i][m][j]}^{p}$ and $F_{a[i][m][j]}^{p}$ are respectively the portfolio discount factor and forward rate falling within the issue interval demarcated by the coupon date $m$ and $m-1$ of issue $i . F_{b[i][m][j]}^{i}$ is the forward rate of an adjacent issue overlapping with the issue interval demarcated by the coupon date $m$ and $m-1$ of issue $i . t_{j}^{j+1}$ refers to the portflio interval, and $t_{l}^{l+1}$ to the adjacent issue interval, and $J^{i m}$ and $L^{i m}$ are respectively the number of portfolio and adjacent issue intervals falling within a particular issue interval demarcated by the coupon date $m$ and $m-1$ of issue $i . C^{i v}, C^{i p d}, C^{i p f d}$, and $C^{i i f d}$ are all different ceiling or maximum values.

From the above, it should be clear that conventional models may find it difficult to implement issue-level term structure decomposition, particularly when including constraints to prevent negative forward rates, and simultaneously maintaining a strict relationship between the issue and portfolio term structures.

\section{Methodology}

To test the model, a market term structure was simulated by selecting a number of intervals, randomly selecting the forward rates of the interval points between a set minimum (0) and maximum (0.3), and interpolating the forward rates of the remaining points by means of straight lines across the intervals. Such a structure may perhaps not be practical. Nevertheless, it poses a difficult term structure to decompose, and may help to illustrate model power.

All government zero coupon and vanilla bonds from the South African market for a given date (2014-07-09) were taken, and discounted by the simulated term structure, to obtain simulated values for these issues, using their original coupon data. The issues' simulated prices and original coupon data were used to decompose a term structure, and the result was compared to the simulated structure. In this case, the minimum issue price modelling error at which structure variance becomes restrictive further lowering or lifting the variance ceiling of equation 6.f no longer accompanies an improvement in modelling error, but rather worsens it is sought.

The given sample constitutes 27 issues, with 211 portfolio coupon dates, resulting in 211 portfolio discount factors.

Marciniak (2006) notes that smoothness of the yield curve may be a key feature if the purpose of analysis is not identification of mispriced securities but rather analysis of general properties and dynamics of the yield curve and extraction of implied expectations regarding inflation, interest rates or future state of the economy. Obviously, also for 
purposes of pricing securities an adequate degree of smoothness is necessary but to a lower degree. Goodness of fit plays a crucial role if interest rate estimates are used for pricing purposes. In order to ensure adequate precision of estimates it is necessary to use a sufficiently flexible curve model. A flexible curve precisely reflecting the current market situation may be a useful tool for identifying optimal maturity segments for a bond issuer or investor. If in a certain market segment a "hump" or a local minimum has emerged and the corresponding yields deviate from their fair values then it may be possible to purchase or sell (issue) a bond under favourable conditions and make extra profits.

In light of this, the model was also run on the original price data of the said government bond sample, for different structure variance ceilings, to note the improvement in modelling error against the change in structure variance.

\section{Analysis}

Figure 1 to 3, and table 1 pertain to the simulated sample. Figure 1 and 2 show the simulated and modelled spot rate and forward rate term structures. Table 1 lists the various modelling residuals, structure variance sums, and corresponding variance ceilings of a number of ceiling values selected in searching for a decomposition with an optimal modelling error viz-a-viz structure variance. The correlation or degree of fit between the simulated and modelled term structure was not quantified. However, modelling residual measures so low, such that it can be viewed as insignificant or essentially zero, and modelling residual fails to be a differentiating factor any longer. A greater number of issues contributing to the portfolio could perhaps have improved the fit even more. Figure 3 shows the issues contributing coupons to the portfolio coupons and thus term structure coefficients, and reflects on the data density per portfolio term structure coefficient.

Figure 4 to 7 , and table 2 pertain to the market sample. Figure 4 shows all decompositions that were done for the market sample, each with a different variance ceiling, and thus modelling error and structure variance. Table 2 lists the variance ceilings, modelling error, structure variance sums, modelling error percentages, and change in variance to change in modelling error ratios of the different simulations. Figure 5 to 7 capture sub-sets of the different decompositions.

The modelling error percentage spans from around $2 \%$ to around $0.4 \%$, with a corresponding span in structure variance of 0.0001 to 55. Around a structure variance of 1 , the improvement in modelling error slows down substantially. The number of sub-structures as well as their magnitude significantly expands as the variance ceiling is lifted, and the modelling error improves. Figure 5 counts two, five and nine peaks and valleys, and figure 6 and 7 count about twenty peaks and valleys. It can also be said that the structure itself shifts as the variance ceiling is lifted, and the modelling error improves. In some cases, the actual position where a sub-structure develops, shifts.

In all decompositions, a residual modelling error persists. Beyond a structure variance of 54.1, a gap starts to form between the structure variance and the variance ceiling. This indicates that the ceiling is no longer that relevant, and in such cases, the decompositions may contain unnecessary variance and this is certainly evident in the case of the decomposition with a variance ceiling of 60 .

Table 1. Residual and variance sums for variance ceilings selected simulated sample

\begin{tabular}{llll}
\hline & Modelling Residual & Variance Sum & Variance Ceiling \\
\hline 1 & $7.2677507164 \mathrm{e}-05$ & $1.2718862337 \mathrm{e}+01$ & $1.5266840874 \mathrm{e}+01$ \\
\hline 2 & $6.0175864292 \mathrm{e}-11$ & $6.2324410009 \mathrm{e}+00$ & $7.6334204369 \mathrm{e}+00$ \\
\hline 3 & $8.0589046547 \mathrm{e}-10$ & $3.0506717597 \mathrm{e}+00$ & $3.8167102184 \mathrm{e}+00$ \\
\hline 4 & $5.3500315289 \mathrm{e}-11$ & $1.5260966662 \mathrm{e}+00$ & $1.9083551092 \mathrm{e}+00$ \\
\hline 5 & $1.4944490090 \mathrm{e}-11$ & $8.2876689407 \mathrm{e}-01$ & $9.5417755461 \mathrm{e}-01$ \\
\hline 6 & $5.4321786510 \mathrm{e}-01$ & $4.7708662669 \mathrm{e}-01$ & $4.7708877730 \mathrm{e}-01$ \\
\hline 7 & $1.3708145730 \mathrm{e}-10$ & $7.4582186419 \mathrm{e}-01$ & $8.2876689407 \mathrm{e}-01$ \\
\hline 8 & $3.7075054138 \mathrm{e}-10$ & $6.9275065159 \mathrm{e}-01$ & $7.4582186419 \mathrm{e}-01$ \\
\hline 9 & $2.1037749320 \mathrm{e}-10$ & $6.5971128322 \mathrm{e}-01$ & $6.9275065159 \mathrm{e}-01$ \\
\hline 10 & $1.1990408666 \mathrm{e}-11$ & $6.3976015629 \mathrm{e}-01$ & $6.5971128322 \mathrm{e}-01$ \\
\hline 11 & $1.9557688802 \mathrm{e}-10$ & $6.2828686451 \mathrm{e}-01$ & $6.3976015629 \mathrm{e}-01$ \\
\hline 12 & $1.6583889817 \mathrm{e}-10$ & $6.2227415332 \mathrm{e}-01$ & $6.2828686451 \mathrm{e}-01$ \\
\hline 13 & $5.5868376592 \mathrm{e}-10$ & $6.1967740580 \mathrm{e}-01$ & $6.2227415332 \mathrm{e}-01$ \\
\hline 14 & $1.0557954511 \mathrm{e}-10$ & $6.1895811593 \mathrm{e}-01$ & $6.1967740580 \mathrm{e}-01$ \\
\hline 15 & $3.1402791478 \mathrm{e}-10$ & $6.1888819114 \mathrm{e}-01$ & $6.1895811593 \mathrm{e}-01$ \\
\hline
\end{tabular}




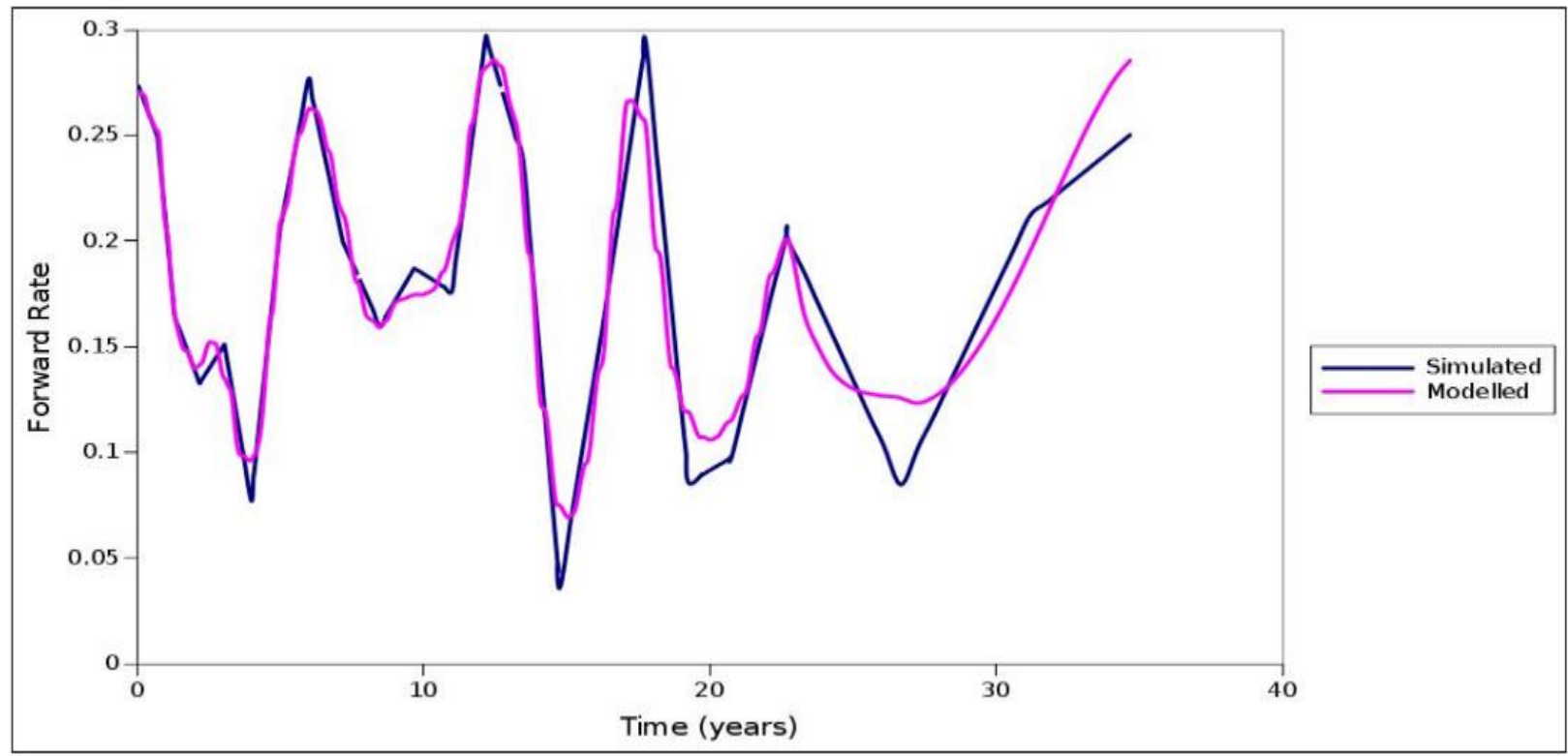

Figure 1. Simulated and modelled forward rate plot

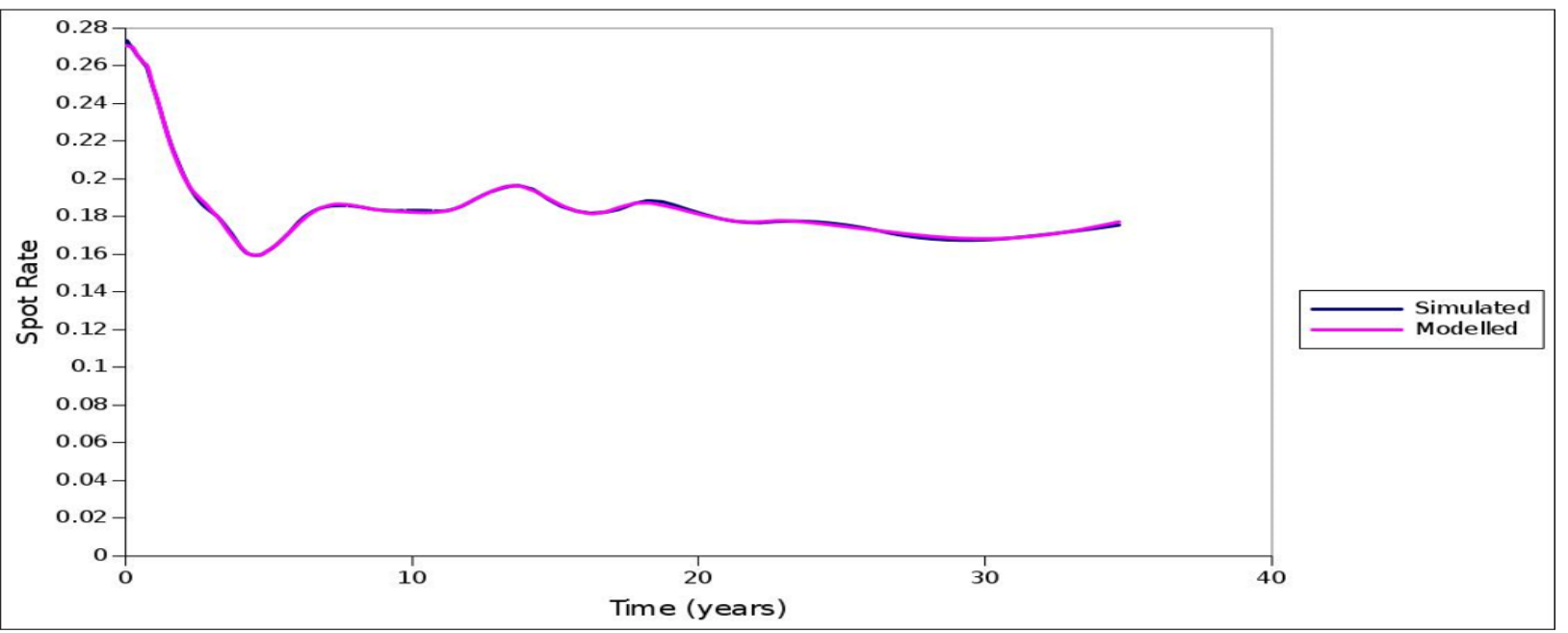

Figure 2. Simulated and modelled spot rate plot

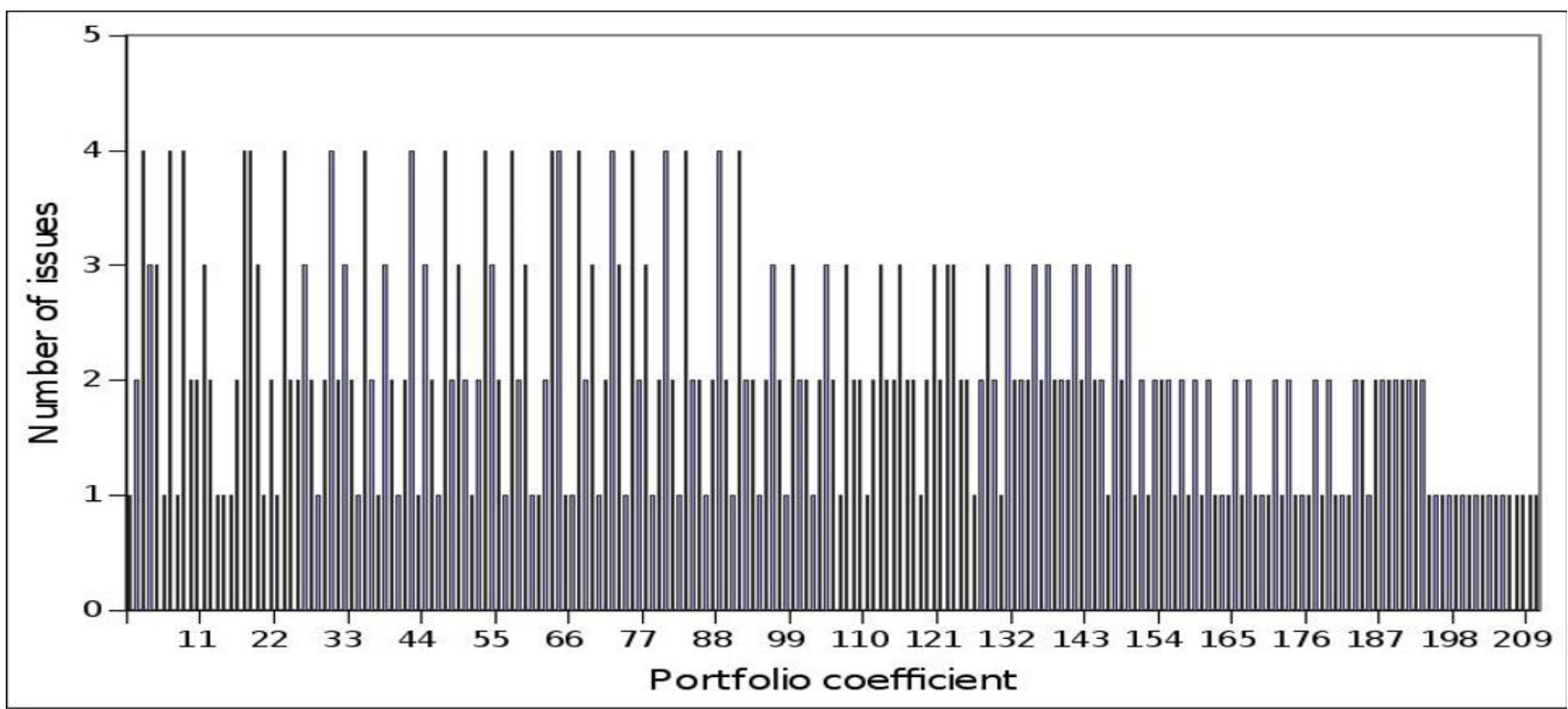

Figure 3. Issues per portfolio coupon date 
Table 2. Residual and variance sums for variance ceilings selected market sample

\begin{tabular}{|c|c|c|c|c|c|}
\hline & $\begin{array}{l}\text { Variance } \\
\text { Ceiling }\end{array}$ & Variance & $\begin{array}{c}\text { Residual (Modelling } \\
\text { Error) }\end{array}$ & Residual \% & $\Delta$ Variance / $\Delta$ Residual \\
\hline 1 & 0.0001 & $1 \mathrm{E}-04$ & 48.7275 & 2.02209 & 487275 \\
\hline 2 & 0.001 & 0.00099 & 24.6952 & 1.0248 & 26872.6 \\
\hline 3 & 0.01 & 0.00999 & 21.5782 & 0.89545 & 346.594 \\
\hline 4 & 0.1 & 0.09996 & 17.7168 & 0.73521 & 42.9152 \\
\hline 5 & 1 & 1 & 12.8663 & 0.53393 & 5.3892 \\
\hline 6 & 2 & 2 & 12.0189 & 0.49876 & 0.84744 \\
\hline 7 & 3 & 3 & 11.7097 & 0.48593 & 0.30914 \\
\hline 8 & 5 & 5 & 11.2856 & 0.46833 & 0.21205 \\
\hline 9 & 10 & 10 & 10.6707 & 0.44281 & 0.12299 \\
\hline 10 & 20 & 20 & 9.89885 & 0.41078 & 0.07718 \\
\hline 11 & 40 & 40 & 9.72896 & 0.40373 & 0.00849 \\
\hline 12 & 50 & 50 & 9.69966 & 0.40252 & 0.00293 \\
\hline 13 & 52 & 52 & 9.69562 & 0.40235 & 0.00202 \\
\hline 14 & 54.1 & 54.1 & 9.6916 & 0.40218 & 0.00192 \\
\hline 15 & 55 & 54.9739 & 9.69145 & 0.40217 & 0.00017 \\
\hline 16 & 60 & 59.7928 & 9.69145 & 0.40217 & $5.3 \mathrm{E}-10$ \\
\hline
\end{tabular}

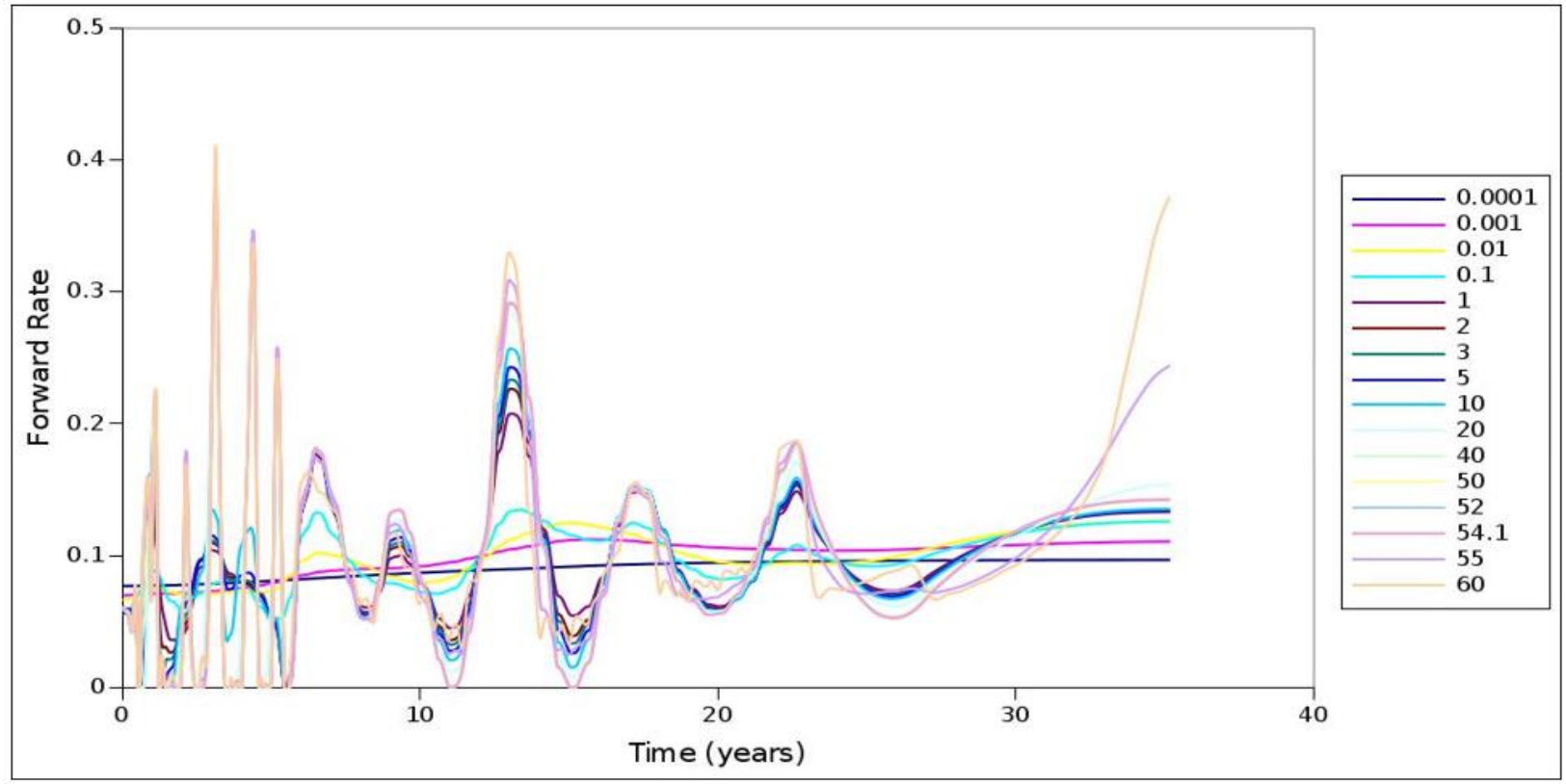

Figure 4. Forward rate term structures (A) 


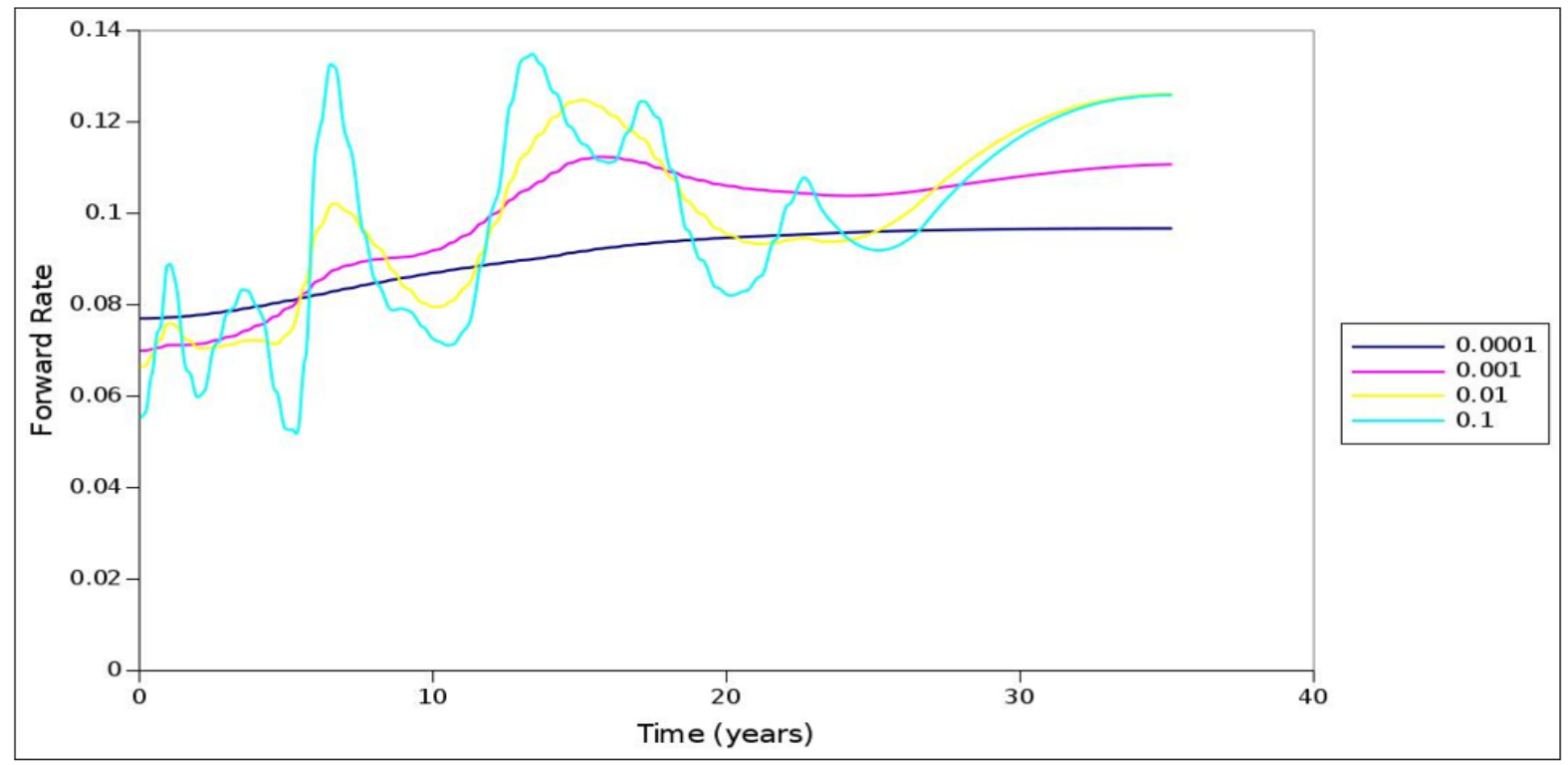

Figure 5. Forward rate term structures (B)

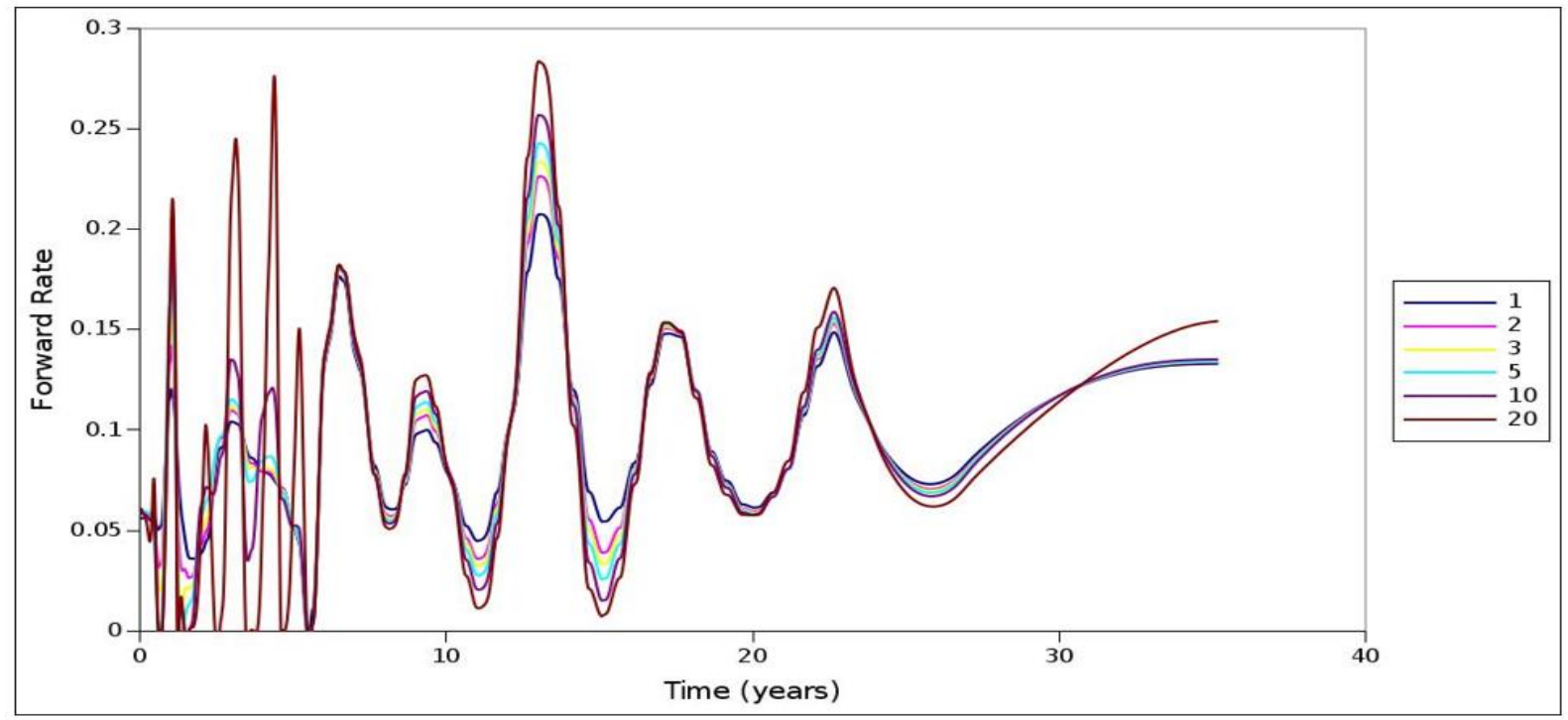

Figure 6. Forward rate term structures (C)

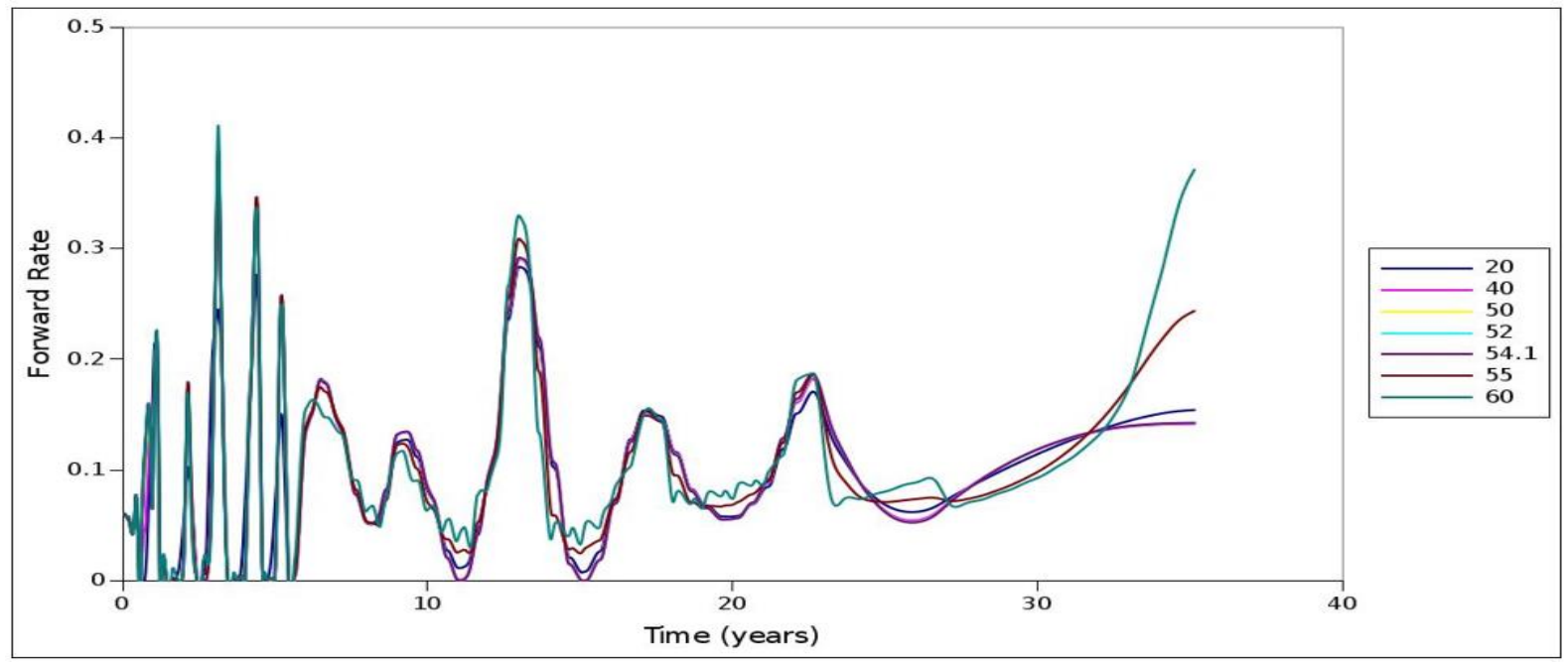

Figure 7. Forward rate term structures (D) 


\section{Conclusion}

Approaching term structure decomposition as an axiomatic structural-based optimization problem is indeed possible. It show promising results in terms of model accuracy or power, based on the modelling error achieved, and the structures that are and can be decomposed. The model achieves above average results in terms of residual modelling error goodness of fit and structure variance or smoothness of the decompositions with low variance ceilings in the range of 0.0001 to 1 have structure smoothness comparable to that of conventional models. Modelling decomposition in this manner has the benefit that it allows great flexibility. Conventional models would find it difficult to match this flexibility through functions or splines.

The greater power of the model may also make it more clear why spline-based models potentially oscillate. At this point it is possible to differentiate between model-induced modelling residuals, in contrast with issue-induced modelling residuals. Model-induced modelling residuals may be introduced by the model or then its underlying requirements particularly in line with obtaining a balance between goodness of fit and smoothness. Issue-induced modelling error refers to the likelihood of unsystematic risk that can not be removed by any model, regardless of its flexibility or power, that consequently remains as a modelling residual. Even when lifting the variance ceiling to a maximum, some modelling error persists, pointing to the existence of a remaining unsystematic risk component.

Without disregarding the argument for structure smoothness, when structure variance is sufficiently overlooked for the moment, it appears that the excess structure variation perceived from the vantage point of acceptable model smoothness may approach individual issue unsystematic risk. In light of this, it is questioned whether it is indeed possible to include pure unsystematic risk components into a term structure. Thus, can the excess structure variation really be seen as unsystematic risk, or should it theoretically be seen as ascribable to systematic risk? The argument that can be made is that, as long as it accompanies an improvement in modelling error, it may be seen as a more efficient loading and representation of systematic risk. In light of this, it is proposed that, with (perceived) poor structure smoothness, what appears as unsystematic risk or simply oscillation may indeed be systematic risk.

If it is not possible to include pure unsystematic risk into a decomposed structure, issue-specific factors and market efficiency may be at play. Bolder and Gusba (2002) and Marciniak (2006) both note the impact of liquidity on market prices and thus decomposition, and Marciniak (2006) also highlights market efficiency in the context of decomposition. Issue-specific factors may contribute both to systematic and unsystematic risk the factors are simultaneously local to the issue, and global in terms of the portfolio. Default risk serves as an example the possibility of default not only affects a particular issue, but all concurrent issues. Thus, factors like liquidity may proxy and reflect on systematic risk factors, like inflation, the risk free rate, and related expectations. It is clear that market efficiency then also plays a role the extent by which expectations and thus issues are efficiently priced. Evidently, greater model power surfaces such issues and anomalies, and may perhaps aid pricing.

Evidently, portfolio-specific factors also have an impact on decomposition. The number of issues of the portfolio may affect maturity structure or spread, and thus make decomposition less sensitive to the individual issues. Better maturity structures should also aid more efficient pricing.

At the same time, evaluating model stability across a number of longitudinal decompositions should help to indicate whether the excess structure variation can be indeed seen as systematic risk. If it is indeed systematic risk, it should also persist across a number of decompositions.

To select a variance ceiling, in order to practically apply the model, a) minimum modelling error (the point where the marginal improvement in modelling error is negligible); b) own discretion; c) the trade-off between residual systematic risk improvement, against the increase in structure variance; d) the structure variance of other models as reference; or e) a similar approach followed by spline-based models' penalty functions and stabilizations, can all be considered. It should also be possible to source a calibrated variance ceiling, by noting the variance ceiling that minimizes structure variance across a number of longitudinal decompositions, similar to a model stability test.

Further attention can be paid to evaluating the model against conventional decomposition models. For this purpose, the goodness-of-fit of the model can be compared against that of conventional models, for the same structure variance measure. The stability of the model across a number of longitudinal decompositions should also receive further attention. Also, it can be further investigated whether the model is easier to apply to markets that find Nelson-Siegel implementations problematic or difficult, and in need of calibration (Gimeno and Pineda, 2006; Gauthier and Simonato, 2012). 


\section{References}

Anderson, N., \& Sleath, J. (2001). New estimates of the UK real and nominal interest rates. Working Paper, No. 126, Bank of England, London.

Bolder, D. J., \& Gusba, S. (2002) Exponentials, Polynomials, and Fourier Series: More Yield Curve Modelling at the Bank of Canada. Bank of Canada Working Paper.

Bolder, D. J., Johnson, G., \& Metzler, A. (2004). An empirical analysis of the canadian term structure of zero-coupon interest rates. Bank of Canada Working Paper.

Boyd, S., \& Vandenberghe, L. (2004). Convex Optimization. Cambridge University Press. https://doi.org/10.1017/CBO9780511804441

Elton, E. J., Gruber, M. J., Agrawal, D., \& Mann, C. (2001). Explaining the Rate Spread on Corporate Bonds.The Journal of Finance, 56(1).

Fisher, M., Nychka, D., \& Zervos, D. (1994). Fitting the Term Structure of Interest Rates with Smoothing Splines. U.S. Federal Reserve Board Working Paper.

Gauthier, G., \& Simonato, J. G. (2012). Linearized Nelson-Siegel and Svensson models for the estimation of spot interest rates. European Journal of Operational Research, 219 (2), 442-451. https://doi.org/10.1016/j.ejor.2012.01.004

Gimeno, R., \& Pineda, J. M. N. (2006). Genetic algorithm estimation of interest rate term structure. Documentos detrabajo del Banco de España, (34), 9-36.

Li, B. E., DeWetering, G., Lucas, R. B., \& Shapiro, A. (2001). “Merrill Lynch Exponential Spline Model”. Merrill Lynch Working Paper.

Marciniak, M. (2006). Yield curve estimation at the National Bank of Poland. Bank $i$ Kredyt, 10, 52-74.

McCulloch, J. H. (1971). Measuring the Term Structure of Interest Rates. Journal of Business, 44, 19-31. https://doi.org/10.1086/295329

McCulloch, J. H. (1975). The Tax-Adjusted Yield Curve. The Journal of Finance, 30(3), 811-830. https://doi.org/10.1111/j.1540-6261.1975.tb01852.x

Nelson, C. N., \& Siegel, A. F. (1987). Parsimonious Modeling of Yield Curves. Journal of Business, 60 (4), $473-489$. https://doi.org/10.1086/296409

Svensson, L. E. (1994). Estimating and interpreting forward interest rates: Sweden 1992-1994 (No. w4871). National bureau of economic research.

Vasicek, O. A., \& H. G. Fong (1981): “Term Structure Modeling Using Exponential Splines”. The Journal of Finance, 37, 339-348. https://doi.org/10.1111/j.1540-6261.1982.tb03555.x

Waggoner, D. F. (1997). Spline Methods for Extracting Interest Rate Curves from Coupon Bond Prices. Working Paper, No. 97-10, Federal Reserve Bank of Atlanta, Atlanta.

\section{Copyrights}

Copyright for this article is retained by the author(s), with first publication rights granted to the journal.

This is an open-access article distributed under the terms and conditions of the Creative Commons Attribution license which permits unrestricted use, distribution, and reproduction in any medium, provided the original work is properly cited. 